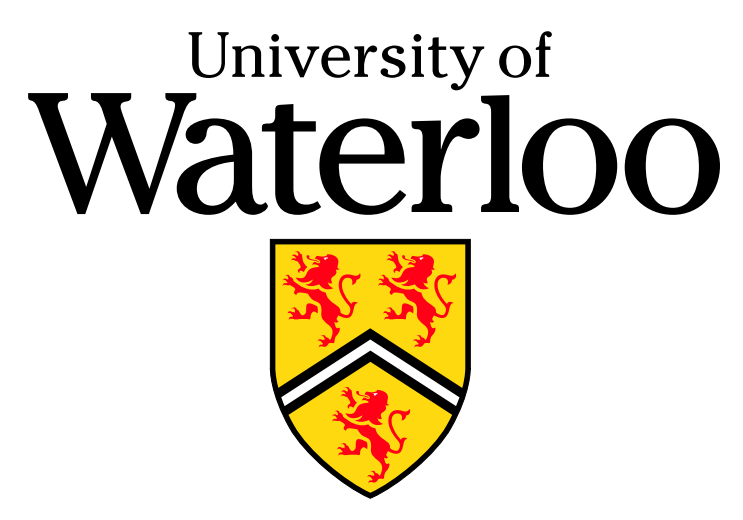

\title{
Delay-Throughput Analysis in Decentralized Single-Hop Wireless Networks
}

by

Jamshid Abouei, Alireza Bayesteh and Amir K. Khandani

Electrical and Computer Engineering Department

University of Waterloo

Waterloo, Ontario, Canada

Email: \{jabouei, alireza, khandani\}@cst.uwaterloo.ca

Technical Report UW-ECE \#2007-04

January, 2007

(C)Jamshid Abouei, Alireza Bayesteh and Amir K. Khandani 2007 


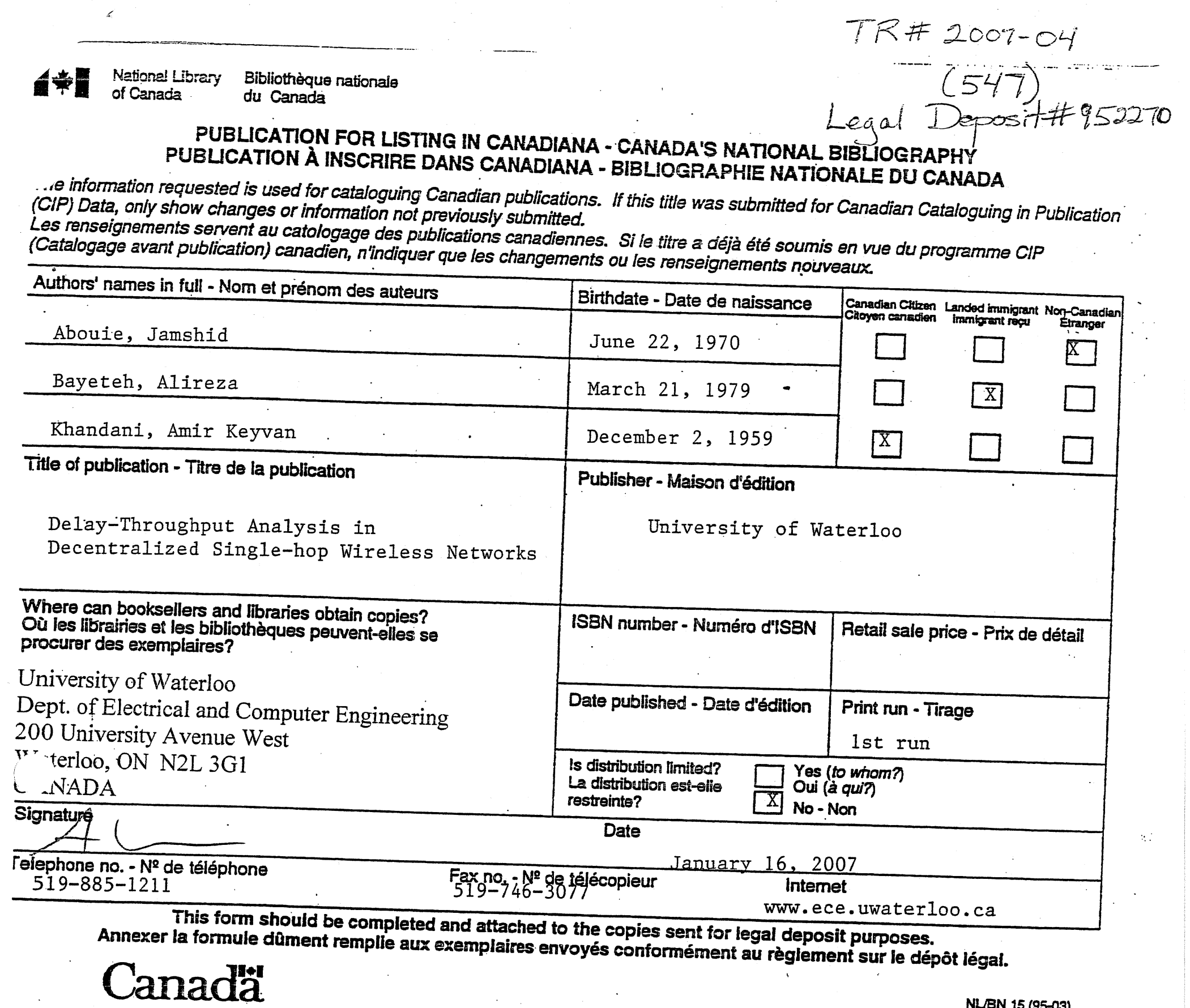



$\begin{array}{ll}\text { Library and Archives } & \begin{array}{l}\text { Bibliothèque et Archives } \\ \text { Canada }\end{array} \\ \text { Canada } & \end{array}$
Canada

\section{RECEIPT FOR LEGAL DEPOSIT REÇU POUR DÉPÔT LÉGAL}

Abouei, Jamshid, 1970-

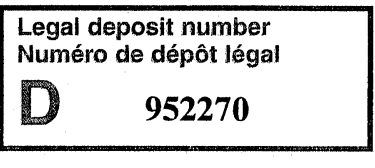

ISBN / ISSN

Delay-throughput analysis in decentralized single-hop wireless networks / by Jamshid Abouei, Alireza Bayesteh and Amir K. Khandani

Waterloo, ON : University of Waterloo. Dept. of Electrical \& Computer Engineering, 2007.

University of Waterloo. Dept. of

Electrical \& Computer Engineering

Price:

Betty Slowinski

200 University AVE W

WATERLOO ON N2L 3G1

Date received:

Date de réception : $\quad$ 2007-01-24

LAC-BAC 0054 (2006/06)

Canadä

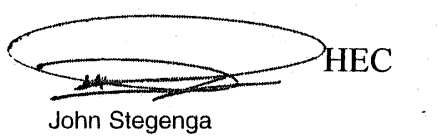




\title{
Delay-Throughput Analysis in Decentralized Single-Hop Wireless Networks
}

\author{
Jamshid Abouei, Alireza Bayesteh and Amir K. Khandani
}

\begin{abstract}
In this paper, an asymptotic analysis for the delay-throughput of a single-hop wireless network with $n$ pairs of nodes is presented. The analysis relies on the decentralized on-off power allocation strategy, in which the on-off transmission policy for each link is based on comparing its direct channel gain with optimum threshold $\tau_{n}$. We first provide a new definition of the transmission delay in a homogenous network. It is proved that the delay threshold level that results the dropping probability for each link tends to zero, while achieving the maximum average sum-rate scales as $\omega(n / \log n)$. Also, the minimum delay in order to make the dropping probability for the whole network approach zero scales as $\omega(n / \log n)+n$. Furthermore, we drive lower and upper bounds for the link activation probability, $q$, such that the order of the average sum-rate is preserved. Based on the upper bound on $q$, an asymptotic analysis shows that the delay in each link and in the network improves without any significant impact on the the average sum-rate. Finally, we present a new definition of the throughput for the link in the cases of one and infinite buffer size. It is demonstrated that the maximum average throughput of the network with the decentralized on-off power allocation strategy is independent of the buffer size.
\end{abstract}

This work is financially supported by Communications and Information Technology Ontario (CITO), Nortel Networks, and Natural Sciences and Engineering Research Council of Canada (NSERC).

The authors are affiliated with the Coding and Signal Transmission Laboratory, Electrical and Computer Engineering Department, University of Waterloo, Waterloo, ON, N2L 3G1, Canada, Tel: 519-884-8552, Fax: 519-888-4338, Emails: \{jabouei, alireza, khandani\}@cst.uwaterloo.ca. 


\section{Index Terms}

Delay, dropping probability, on-off power allocation, single-hop wireless network, throughput.

\section{INTRODUCTION}

As the demand for higher data rates increases, effective resource allocation emerges as the main issue in wireless networks to satisfy quality of service (QoS) requirements. Among various resource allocation schemes, the decentralized power control algorithms for maximizing the throughput have attracted significant attention [1]-[3]. Also, lower transmission delay is an important QoS requirement in bufferlimited networks. Particularly, for backlogged users $^{1}$ with real-time services (e.g., interactive games, live sports videos, etc), too much delay results in dropping some packets. Therefore, the main challenge in real-time services is to utilize an efficient power allocation scheme such that the delay is minimized while achieving a high throughput.

The throughput maximization of the cellular and multihop wireless networks has been extensively studied in [4]-[8]. In these works, no delay analysis is performed. However, it is shown that the high throughput in the network is achieved at the cost of a high amount of delay. This problem has motivated the researchers to study the relation between the delay characteristics and the throughput. Particularly, in most recent literature [9]-[15], the tradeoffs between delay and throughput have been investigated as a key measure of the network performance. For instance, Sharif and Hassibi [9] analyze the delay characteristics and the throughput in broadcast channel networks. They propose an algorithm to reduce the delay without too much degradation in the throughput. The first studies on achieving a high throughput and low delay in ad-hoc wireless networks are framed in [10] and [11]. This line of work is further expanded in [12]-[14] by using different mobility models such as the random walk and the Brownian mobility models. El Gamal et al. [12] analyze

\footnotetext{
${ }^{1}$ For each user, there is always a packet available to be transmitted.
} 
the optimal delay-throughput scaling for different of wireless network topologies. In the static random network with $n$ nodes, they prove that the optimal tradeoff between throughput $T_{n}$ and delay $D_{n}$ is given by $D_{n}=\Theta\left(n T_{n}\right)$. They also show that the same result is achieved in random mobile networks, when $T_{n}=O(1 / \sqrt{n \log n})$. Neely and Modiano [14] consider the delay-throughput tradeoff only for mobile ad-hoc networks. They have investigated the delay characteristics by using the redundant packets transmission through multiple paths.

In this paper, we present the delay-throughput analysis in a single-hop wireless network with $n$ links. Also, we use the decentralized on-off power allocation strategy proposed in [3], in which the transmission policy for link $i$ is to compare the direct channel gain $h_{i i}$ with optimum threshold $\tau_{n}$. Our analysis is different from the delay analysis with the ON/OFF Bernoulli scheme in [16]. Primarily, we utilize a decentralized approach with local information, while [16] considers a central controller to study the channel conditions of all the links in the system. Unlike [8], in our algorithm, we do not use relay nodes. The channel model that we use in the network is quasi-static Rayleigh fading. This is different from the geometric models in [12]-[14] that is based on the distance between the sources and the destinations.

Assuming user $i$ knows only its direct channel gain, $h_{i i}$, the optimum threshold $\tau_{n}$ is chosen such that the average sum-rate is maximized. It is shown that increasing the number of links gives rise to increasing the average sum-rate, at the cost of increasing the delay. This results in higher packet droppings in the real-time applications with limited buffer sizes. To study the delay-throughput tradeoff, we first provide a new definition of the delay. Also, we present the delay characteristics from the link and for the network points of view. It is proved that the minimum delay in order to make the dropping probability for each link and the whole network tend to zero scale as $\omega(n / \log n)$ and $\omega(n / \log n)+n$, respectively. Also, we address the question: How can we achieve a better delay performance without sacrificing too much the throughput? It is demonstrated that the tradeoff between the delay and the average sum-rate is strongly influenced by the threshold level $\tau_{n}$ that depends on the channel model. We 
show that by relaxing the value of $\tau_{n}$, the delay is improved without changing the order of the average sum-rate. We further present a new definition of the throughput for one buffer size and infinite backlogged user.

The rest of the paper is organized as follows. In Section II, the network model is introduced. The on-off power allocation strategy is presented in Section III. The delay characteristics in terms of the dropping probability are analyzed in Section IV. In Section V, we establish the delay-throughput tradeoff for the network. Finally, in Section VI, an overview of the results and conclusions is presented, and directions for ongoing and future research are mentioned.

Knuth's notation [17]: For any functions $f(n)$ and $g(n)$ :

- $f(n)=O(g(n))$ means that $\lim _{n \rightarrow \infty}|f(n) / g(n)|<\infty$.

- $f(n)=\Omega(g(n))$ means that $\lim _{n \rightarrow \infty}|f(n) / g(n)|>0$.

- $f(n)=o(g(n))$ means that $\lim _{n \rightarrow \infty}|f(n) / g(n)|=0$.

- $f(n)=\omega(g(n))$ means that $\lim _{n \rightarrow \infty}|f(n) / g(n)|=\infty$.

- $f(n)=\Theta(g(n))$ means that $\lim _{n \rightarrow \infty}|f(n) / g(n)|=c$, where $0<c<\infty$.

Throughout the paper, we use $\mathbb{N}_{n}$ for representing the set $\{1,2, \ldots, n\}$ and $\log ($. as the natural logarithm function.

\section{NeTWORK MODEL}

Consider a single-hop wireless network, in which $n$ pairs of nodes ${ }^{2}$, indexed by $1, \ldots, n$, are located within the network area (Fig. 1). We assume the number of links, $n$, is a common information between the nodes. All the nodes in the network are assumed to have a single antenna. Also, it is assumed that all the transmissions occur over the same bandwidth. The time axis is divided into slots with the duration of one block. The slot duration is assumed to be equal for all the links. Here, we assume a buffer limited network, where each link has the buffer size equal to one packet. Also, we consider a homogeneous network, in which all the links have the

\footnotetext{
${ }^{2}$ In this work, the term "pair" is used for the transmitter and the related receiver, and "user" only for the transmitter.
} 


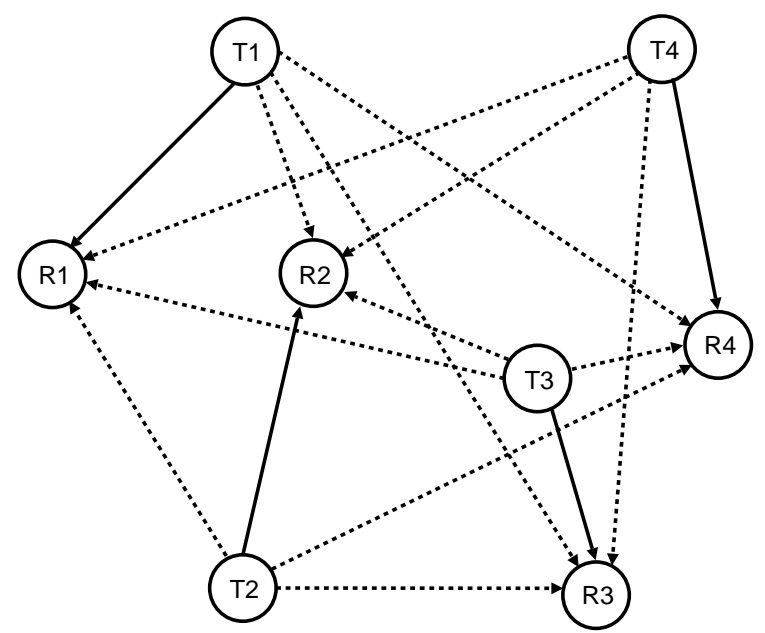

Fig. 1. A single-hop wireless network with $n=4$.

same configurations and use the same protocols. Thus, the transmission strategy for all nodes are agreed in advance.

The channel is supposed to be quasi-static Rayleigh fading. In this model, the channel gains remain constant while transmitting one block and change independently from block to block. The link between transmitter $j$ and receiver $i$ has the channel gain $h_{j i}=\left|g_{j i}\right|^{2}$, where the complex variables $g_{j i}$ 's are the channel coefficients. Under a Rayleigh fading channel condition, $h_{j i}$ 's are exponentially distributed with unit mean. Here, we assume that each receiver knows only its own direct channel gain at the beginning of each block. This Channel-State Information (CSI) is fed back to the transmitter in a dedicated zero-delay and error-free channel.

We denote the average transmit power of user $i$ by $p_{i} \in[0,1]$. Also, the nonnegative vector $\mathbf{P}=\left(p_{1}, \ldots, p_{n}\right)$ represents the vector of all the users' powers in the network. The power of Additive White Gaussian Noise (AWGN) in each receiver is assumed to be $N_{0}$. The interference from the other users on receiver $i$ is equal to

$$
I_{i}\left(\mathbf{P}_{-i}\right)=\sum_{\substack{j \neq i \\ j=1}}^{n} h_{j i} p_{j},
$$

where $\mathbf{P}_{-i}=\left(p_{1}, \ldots, p_{i-1}, p_{i+1}, \ldots, p_{n}\right)$. Assuming Gaussian signal transmission from all 


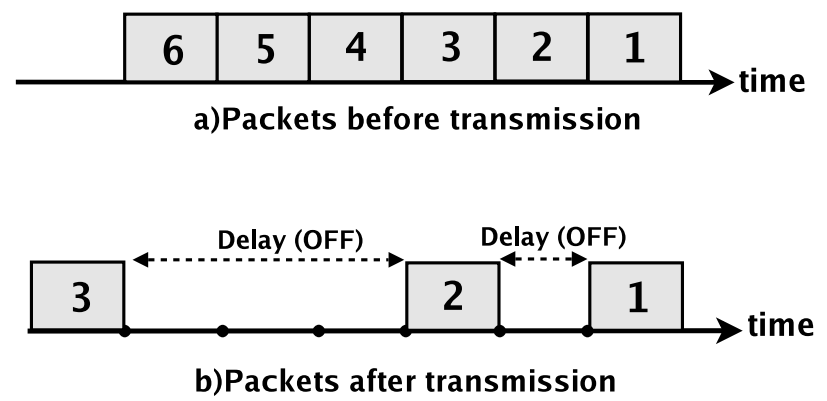

Fig. 2. Queueing model for the on-off power allocation strategy.

the transmitters, the distribution of the interference will be Gaussian as well. Thus, the signal-to-interference-plus-noise ratio (SINR) of link $i$ is defined as

$$
\gamma_{i}(\mathbf{P}) \triangleq \frac{h_{i i} p_{i}}{I_{i}\left(\mathbf{P}_{-i}\right)+N_{0}} .
$$

The SINR determines the achievable data rate of each link as follows:

$$
R_{i}(\mathbf{P})=\log \left(1+\gamma_{i}(\mathbf{P})\right)
$$

We assume the packet arrival rate is the same for all the links, and is equal to $1 / \lambda$ packets per block length. Also, we define the random variable $D_{i}$ for link $i$ as the latency between two successive transmissions, expressed as the number of blocks (see Fig. 2). In this work, we consider the packet dropping probability as a performance criterion in the delay sensitive applications. Dropping occurs when the latency between two successive transmissions in each link exceeds a prespecified level. In fact, since the buffer size is one and the packets are arrived with a constant rate $1 / \lambda$, it follows that the dropping occurs when $D_{i}>\lambda, i \in \mathbb{N}_{n}$. In order to analyze the dropping probability, we define $\lambda_{\ell}$ and $\lambda_{n}$ as the delay threshold levels for the link and for the network, respectively. In this case, we have the following definition.

Definition 1: Let $\mathscr{B}_{i}$ be the event that the dropping occurs in link $i$. Then, the packet dropping probability in the link is defined as

$$
P\left(\mathscr{B}_{i}\right) \triangleq \operatorname{Pr}\left(D_{i}>\lambda_{\ell}\right), \quad \forall i \in \mathbb{N}_{n}
$$


Also, if $\mathscr{B}_{\mathcal{N}}$ represents the event of the packet dropping, then

$$
P\left(\mathscr{B}_{\mathcal{N}}\right) \triangleq \operatorname{Pr}\left(\bigcup_{i=1}^{n}\left(D_{i}>\lambda_{n}\right)\right),
$$

where $P\left(\mathscr{B}_{\mathcal{N}}\right)$ is the packet dropping probability in the network.

\section{ON-OFF Power Scheme}

In this paper, we assume that all the links perform the on-off power allocation strategy proposed in [3]. In this scheme, the transmission rule for each link is to compare its direct channel gain with a prespecified threshold level $\tau_{n}$, i.e.,

$$
p_{i}=\mathcal{F}\left(h_{i i}\right)= \begin{cases}1, & \text { if } h_{i i}>\tau_{n}, \\ 0, & \text { Otherwise }\end{cases}
$$

for all $i \in \mathbb{N}_{n}$. In this strategy, each user transmits with full power or remains silent, independent of the other links and without using the central node. Thus, the power distribution of each user is a Bernoulli random variable with parameter $q$, i.e.,

$$
f\left(p_{i}\right)= \begin{cases}q, & p_{i}=1 \\ 1-q, & p_{i}=0 .\end{cases}
$$

Also, the number of active links is a binomial random variable with parameters $n$ and $q$. Here, we denote $q$ as the probability of the link activation that is defined as $q \triangleq \operatorname{Pr}\left\{h_{i i}>\tau_{n}\right\}$. It should be noted that $\tau_{n}$ is a function of the network size, $n$, and also depends on the channel model. As we see later, the performance of the network is strongly influenced by the choice of $\tau_{n}$.

In this work, we use the average sum-rate as a key measure in the network performance, where the average is computed with respect to $h_{i i}$ 's and $I_{i}$ 's, $i \in \mathbb{N}_{n}$. Letting $\bar{R}_{\text {sum }}$ denote the average sum-rate of the network, we have

$$
\bar{R}_{\text {sum }} \triangleq \mathbb{E}\left[\sum_{i=1}^{n} R_{i}\right]=\sum_{i=1}^{n} \mathbb{E}\left[R_{i}\right] .
$$

Next, we summarize some of our earlier results on the on-off power allocation strategy in single-hop wireless networks reported in [3]. 
Lemma 1: In a network with the on-off power allocation strategy and large $n$, the optimum $\tau_{n}$ that maximizes the average sum-rate scales as

$$
\tau_{n}^{*} \approx \log n-\log \log n+\Theta(1) .
$$

Noting that under a Rayleigh fading channel condition, the probability of the link activation is $q=\operatorname{Pr}\left\{h_{i i}>\tau_{n}\right\}=e^{-\tau_{n}}$, we have the following corollary.

Corollary 1: For the proposed strategy with the optimum threshold $\tau_{n}^{*}$, the probability of the link activation scales as $\Theta\left(\frac{\log n}{n}\right)$.

Corollary 2: In the network with $n$ pairs of nodes, if $m$ is the number of active links, then $\mathbb{E}[m]=n e^{-\tau_{n}} \sim \Theta(\log n)$.

Lemma 2: In the network with the on-off power allocation strategy and the optimum $\tau_{n}^{*}$, the average sum-rate is asymptotically

$$
\begin{aligned}
\bar{R}_{\text {sum }} & =n \mathbb{E}\left[R_{i}\right] \approx 1-\log q \\
& =\log n-\log \log n+\Theta(1) .
\end{aligned}
$$

Interestingly, Lemma 2 implies that the average sum-rate scales as $\Theta(\log n)$, without using the relay and coordination between links. Also, from (10) and using $q=e^{-\tau_{n}}$, it is concluded that the average sum-rate is a function of threshold level $\tau_{n}$. We use this result to analyze the packet dropping probability in the next section.

\section{DElay AnALysis}

In this section, we analyze the delay characteristics in terms of the dropping probabilities for the link and for the whole network. Under the on-off power allocation policy, the transmission traffic for each user is modeled as a two-state discrete-time Markov model shown in Fig. 3. According to this model, the latency $D_{i}$ is a geometric random variable with the probability mass function $\operatorname{Pr}\left\{D_{i}=d\right\}=q(1-q)^{d-1}$.

Lemma 3: In the network with the on-off power scheme, the dropping probabilities of the packets in the link and the network can be obtained as

$$
P(\mathscr{B})=(1-q)^{1+\lambda_{\ell}}
$$




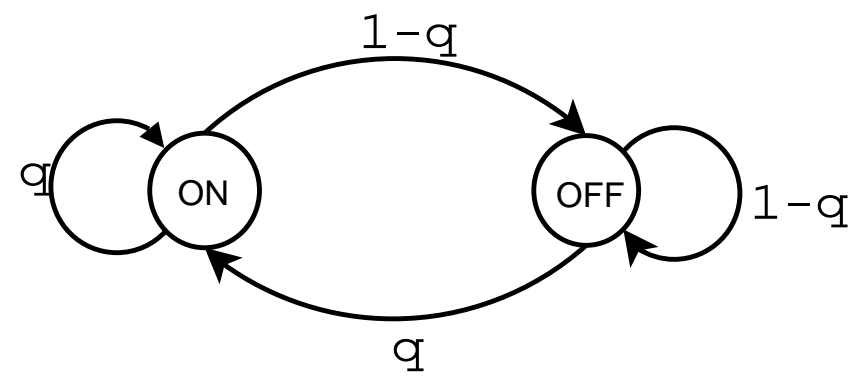

Fig. 3. Discrete-time Markov model for the on-off power allocation strategy.

and

$$
P\left(\mathscr{B}_{\mathcal{N}}\right)=1-(1-P(\mathscr{B}))^{n}
$$

respectively.

Proof: Using (4) and by taking account of the geometric distribution of $D_{i}$, the packet dropping probability in link $i$ is obtained as

$$
\begin{aligned}
P\left(\mathscr{B}_{i}\right) & =1-\operatorname{Pr}\left(D_{i} \leq \lambda_{\ell}\right) \\
& =1-\sum_{y=0}^{\lambda_{\ell}} q(1-q)^{y} .
\end{aligned}
$$

It should be noted that (15) is valid only for $q<1$. In the case of $q=1$, i.e., all the links transmit with full power, no delay exists in each link and consequently $P\left(\mathscr{B}_{i}\right)=0$. Considering the following expression

$$
\sum_{k=0}^{M} x^{k}=\frac{1-x^{M+1}}{1-x}, \quad|x|<1,
$$

the dropping probability of link $i$ is obtained as

$$
P\left(\mathscr{B}_{i}\right)=(1-q)^{1+\lambda_{\ell}}, \quad i=1, \ldots, n .
$$

Since (16) is the same for all the links, we drop the index $i$ and this completes the proof of the first part of the lemma. 
To drive $P\left(\mathscr{B}_{\mathcal{N}}\right)$, let $\mathscr{E}$ denote the occurrence of no dropping event in the network. Thus,

$$
P(\mathscr{E})=\operatorname{Pr}\left\{\mathscr{B}_{1}^{c} \cap \mathscr{B}_{2}^{c} \cap \ldots \cap \cap \mathscr{B}_{n}^{c}\right\}
$$

Noting that the events $\mathscr{B}_{i}^{c}$ and $\mathscr{B}_{j}^{c}$ are independent of each other for all $i, j \in \mathbb{N}_{n}$, we have

$$
\begin{aligned}
P(\mathscr{E}) & =\prod_{k=1}^{n} \operatorname{Pr}\left\{\mathscr{B}_{k}^{c}\right\} \\
& =\prod_{k=1}^{n}\left(1-P\left(\mathscr{B}_{k}\right)\right) \\
& =(1-P(\mathscr{B}))^{n} .
\end{aligned}
$$

Consequently, the dropping probability of the packet in the network is computed as

$$
P\left(\mathscr{B}_{\mathcal{N}}\right)=1-(1-P(\mathscr{B}))^{n}
$$

We are now ready to prove the main result of this section. In the next theorem, we drive the optimum threshold $\lambda_{\ell}$ and $\lambda_{n}$ such that the dropping probabilities in the link and the network tend to zero.

Theorem 1: For the network with the on-off power allocation strategy and the optimum $q$ that maximizes the average sum-rate,

i) $\lim _{n \rightarrow \infty} P(\mathscr{B})=0$, if $\lambda_{\ell} \sim \omega(n / \log n)$.

ii) $\lim _{n \rightarrow \infty} P\left(\mathscr{B}_{\mathcal{N}}\right)=0$, if $\lambda_{n} \sim \omega(n / \log n)+n$.

Proof: i) From (12) and using the Taylor series expansion

$$
\log (1-z)=-\sum_{k=1}^{\infty} z^{k} / k, \quad|z|<1
$$

we have

$$
\begin{aligned}
P(\mathscr{B}) & =e^{\left(1+\lambda_{\ell}\right) \log (1-q)} \\
& =e^{-\left(1+\lambda_{\ell}\right) \sum_{k=1}^{\infty} q^{k} / k}
\end{aligned}
$$


where $q<1$. Since for sufficiently large $n, q \ll 1$, we can approximate (19) as

$$
P(\mathscr{B}) \approx e^{-q\left(1+\lambda_{\ell}\right)}
$$

Setting $q \lambda_{\ell}=\omega(1)$ makes $e^{-q \lambda_{\ell}} \rightarrow 0$. By using the optimum $q$ in Corollary 1 , if $\lambda_{\ell} \sim \omega(n / \log n)$, then $P(\mathscr{B})=o(1)$, i.e.,

$$
\lim _{n \rightarrow \infty} P(\mathscr{B})=0 \text {. }
$$

ii) From (18) and considering the following binomial series

$$
(1-x)^{k}=1-k x+\frac{k(k-1)}{2 !} x^{2}-\ldots
$$

the dropping probability in the network will be

$$
P\left(\mathscr{B}_{\mathcal{N}}\right)=n P(\mathscr{B})-\frac{n(n-1)}{2 !} P^{2}(\mathscr{B})+\ldots
$$

If $\lambda_{n}$ is the optimum threshold such that $\lim _{n \rightarrow \infty} P\left(\mathscr{B}_{\mathcal{N}}\right)=0$, it guarantees a sufficiently small value for $P(\mathscr{B})$ as well. Hence, $(21)$ can be approximated by

$$
P\left(\mathscr{B}_{\mathcal{N}}\right) \approx n P(\mathscr{B})
$$

Similar to the proof of part (i), the dropping probability of the network can be written as

$$
\begin{aligned}
P\left(\mathscr{B}_{\mathcal{N}}\right) & \approx n(1-q)^{1+\lambda_{n}} \\
& \approx e^{-q\left(1+\lambda_{n}\right)+\log n} .
\end{aligned}
$$

Setting $q \lambda_{n}-\log n=\omega(1)$ makes $e^{-q\left(1+\lambda_{n}\right)+\log n} \rightarrow 0$. Thus, for the network with the optimum $q$, choosing $\lambda_{n} \sim \omega(n / \log n)+n$ yields $\lim _{n \rightarrow \infty} P\left(\mathscr{B}_{\mathcal{N}}\right)=0$.

\section{A Study of Delay-Throughput Tradeoffs}

Previously, we investigated the delay based on achieving a minimum dropping probability, while achieving the maximum average sum-rate. In this section, we present some results on improving the delay without any significant impact on the throughput of the network. First, we study the scaling law of the average sum-rate 
with the on-off power scheme. Recall that the optimum value for $q$ that achieves the maximum average sum-rate scales as $\log n / n$. Clearly, increasing the number of the links has the advantage of increasing $\bar{R}_{\text {sum }}$. However, due to decreasing the value of $q$, delay increases. The problem is crucial in the static networks with immobile nodes. Particularly, the links in the bad channels experience too much delay. To decrease the delay, one solution is to assign other frequency bands for the links experiencing bad channel conditions. This could be accomplished by using a central controller to provide fairness between the links. Also, we can use relay hops to reduce the delay. Deploying multiple antennas [18] can improve the delay as well. The other solution that we investigate in this work is to relax the optimum value of threshold $\tau_{n}^{*}$.

\section{A. Delay Improvement}

By using Corollary 2 and noting that $\tau_{n}=-\log q$, reducing $\tau_{n}$ will increase $q$ and the number of the active links. Clearly, this reduces the delay in the network, however according to (10), the average sum-rate decreases as well. Therefore, the threshold $\tau_{n}$ is interpreted as a compromise between delay and average sum-rate. In the following, we drive lower and upper bounds on $\tau_{n}$ such that the order of the average sum-rate is preserved, i.e.,

$$
\lim _{n \rightarrow \infty} \frac{\bar{R}_{\text {sum }}}{\log n}=1 .
$$

Theorem 2: For the network with the on-off power allocation strategy, the average sum-rate is asymptotically of $\operatorname{order} \log n$, if

$$
\Theta\left(\frac{\log n}{n}\right) \leq q \leq \frac{\Lambda(n)}{n},
$$

where $\Lambda(n) \sim \Omega(\log n)$ and satisfies $\Lambda(n)=e^{o(\log n)}$.

Proof: For the lower bound, the theorem is easily proved by using the Corollary 1. Substituting $q=\frac{\Lambda(n)}{n}$ in (10) yields

$$
\bar{R}_{\text {sum }}=1+\log n-\log \Lambda(n) .
$$


To achieve $\bar{R}_{\text {sum }}$ of order $\log n$, the function $\Lambda(n)$ must satisfy

$$
\log \Lambda(n)=o(\log n)
$$

and this completes the proof of the theorem.

Corollary 3: The lower and upper bounds on $\tau_{n}$ that result in $R_{\text {sum }}$ of order $\Theta(\log n)$ are

$$
\log n-\log \Lambda(n) \lesssim \tau_{n} \lesssim \log n-\log \log n+\Theta(1) .
$$

An interesting insight provided by the upper bound in (23) is the improvement of the threshold levels $\lambda_{\ell}$ and $\lambda_{n}$, without changing the order of the average sum-rate.

Corollary 4: The optimum values of $\lambda_{\ell}$ and $\lambda_{n}$ that result in $P(\mathscr{B})$ and $P\left(\mathscr{B}_{\mathcal{N}}\right)$

tend to zero, while achieving $\bar{R}_{\text {sum }}$ of order $\log n$ decrease as $\lambda_{\ell}=\omega\left(\frac{n}{\Lambda(n)}\right)$ and $\lambda_{n}=\omega\left(\frac{n}{\Lambda(n)}\right)+\frac{n \log n}{\Lambda(n)}$, respectively.

Fig. 4-a illustrates $\lambda_{\ell}$ versus the number of links for $\Lambda(n)=\log n$ and $\Lambda(n)=$ $e^{\sqrt{\log n}}$. Also, Fig. 4-b shows the corresponding average sum-rate (24) versus the number of links. From these figures, it is observed that the delay decreases without any significant impact on the average sum-rate.

\section{B. Throughput Maximization}

In this section, we present a new definition of the average throughput for the backlogged users. Also, we derive the maximum average throughput in two extreme cases of one and infinite buffer size. For the network with the on-off power allocation strategy, the average throughput of link $i$ is defined on a per-block basis as

$$
T_{i}=\lim _{L \rightarrow \infty} \frac{1}{L} \sum_{t=1}^{L} R_{i}^{(t)} \mathcal{I}_{i}^{(t)},
$$

where $\mathcal{I}_{i}^{(t)}$ is an indicator variable which is equal 1 , if the user $i$ transmits with full power at block $t$, and 0 otherwise, and $R_{i}^{(t)}$ is the transmission rate at block $t$.

Theorem 3: For the backlogged users with a buffer size of one, the maximum average throughput asymptotically scales as $1 / n$. 


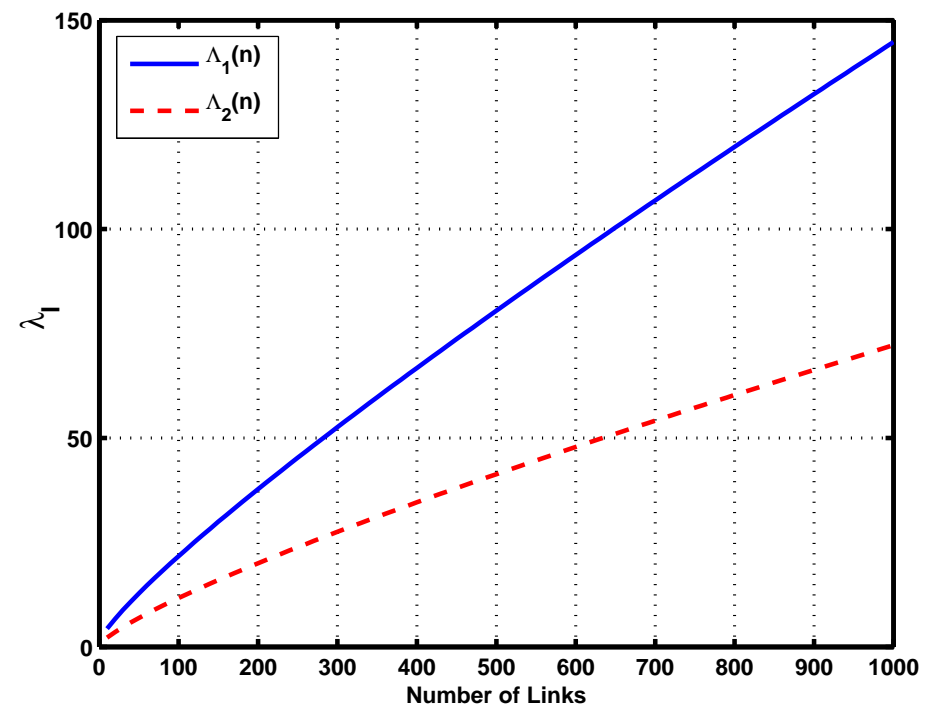

(a)

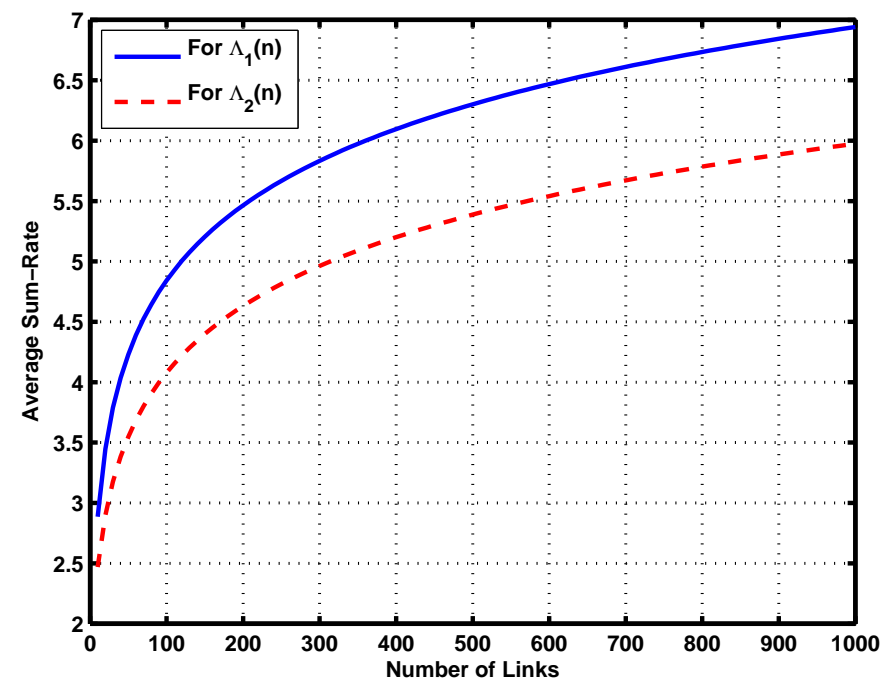

(b)

Fig. 4. a) $\lambda_{\ell}$, and b) Average sum-rate vs. the number of links for $\Lambda_{1}(n)=\log n$ and $\Lambda_{2}(n)=e^{\sqrt{\log n}}$. 
Proof: Let $\epsilon_{\ell}$ denote the blocking probability of the link with a buffer size of one. Noting that the arrival rate of the packets is constant and equal to $1 / \lambda_{\ell}$, where $\lambda_{\ell}$ is obtained as (20), it follows that one packet is transmitted with the probability $1-\epsilon_{\ell}$ during $\lambda_{\ell}$ blocks. Thus, from (26), it is concluded that

$$
\mathcal{I}_{i}^{(t)}= \begin{cases}1, & \text { w. p. } \frac{1-\epsilon_{\ell}}{\lambda_{\ell}} \\ 0, & \text { Otherwise. }\end{cases}
$$

Consequently, the average throughput of the link with a buffer size of one is simplified as

$$
T_{i}=\frac{\mathbb{E}\left[R_{i}\right]}{\lambda_{\ell}}\left(1-\epsilon_{\ell}\right)
$$

According to Lemma 2, we have

$$
\mathbb{E}\left[R_{i}\right]=\frac{\bar{R}_{\text {sum }}}{n}=\frac{1-\log q}{n} .
$$

Also, using (20), it yields

$$
\lambda_{\ell}=\frac{1}{q} \log \frac{1}{\epsilon_{\ell}}-1
$$

Thus,

$$
T_{i}=\frac{1-\log q}{n\left(\frac{1}{q} \log \frac{1}{\epsilon_{\ell}}-1\right)}\left(1-\epsilon_{\ell}\right) .
$$

It is easy to verify that (31) is a positive monotically increasing function of $q$. Thus, the average throughput (31) attains its maximum value at $q=1$. As mentioned in Lemma 3, for $q=1$, there is no packet dropping and as a result the blocking probability is zero, i.e., $\epsilon_{\ell}=0$. Thus, $\lambda_{\ell}=1$ and consequently from (28), we have

$$
T_{i}=\frac{1}{n}
$$

In the next theorem, we obtain the maximum average throughput of the infinite backlogged user. 
Theorem 4: For the backlogged user with infinite buffer size, the optimum solution for the optimization problem

$$
q^{*}=\arg \max _{q} T_{i}
$$

is 1 . Furthermore,

$$
T_{i, \max }=\frac{1}{n}
$$

Proof: For infinite buffer size and from (26), it is concluded

$$
\mathcal{I}_{i}^{(t)}= \begin{cases}1, & q, \\ 0, & 1-q .\end{cases}
$$

Since, $\mathcal{I}_{i}^{(t)}$ is a Bernoulli random variable with parameter $q$, we have $\mathbb{E}\left\{\mathcal{I}_{i}^{(t)}\right\}=q$. Thus, the average throughput defined in (26) is obtained as

$$
\begin{aligned}
T_{i} & =\mathbb{E}\left[R_{i}\right] \mathbb{E}\left[\mathcal{I}_{i}^{(t)}\right] \\
& =\frac{q(1-\log q)}{n}
\end{aligned}
$$

It can be shown that (36) attains its maximum value at $q=1$. Thus, by using (36), the maximum average throughput in the case of infinite buffer size is

$$
T_{i, \max }=\frac{1}{n}
$$

Interestingly, Theorem 4 indicates the maximum average throughput of the network with the decentralized on-off power allocation strategy is independent of the buffer size. Therefore, we can reduce the hardware complexity while achieving the maximum average throughput.

\section{CONCLUSION}

In this paper, we have studied the delay-throughput analysis in a single-hop wireless network with the decentralized on-off power power allocation strategy. We have proved that the delay threshold level that results the dropping probability for each link tends to zero, while achieving the maximum average sum-rate scales as 
$\omega(n / \log n)$. Also, the minimum delay in order to make the dropping probability for the whole network approach zero scales as $\omega(n / \log n)+n$. We have also shown that by relaxing the value of threshold $\tau_{n}$, the delay is significantly improved without changing the order of the average sum-rate. We have presented a new definition for the throughput and derived the maximum throughput in the cases of one and infinite buffer size. It has been shown that the maximum average throughput of the network with the decentralized on-off power allocation strategy is independent of the buffer size.

Throughout this work, it is assumed that all the links use a single antenna and also all the transmissions occur over the same bandwidth. A possible future extension of this work would be to analyze the delay-throughput, when other frequency bands are assigned for the links experiencing bad channel conditions. Also, deploying multiple antennas [18] is another extension.

\section{REFERENCES}

[1] C. U. Saraydar, N. B. Mandayam, and D. J. Goodman, "Efficient power control via pricing in wireless data networks," IEEE Trans. on Commun., vol. 50, no. 2, pp. 291-303, Feb. 2002.

[2] R. Etkin, A. Parekh, and D. Tse, "Spectrum sharing for unlicensed bands," in IEEE Dyspan, Baltimore MD, 2005.

[3] J. Abouei, M. Ebrahimi, and A. K. Khandani, "A new decentralized power allocation strategy in singlehop wireless networks," Technical report, University of Waterloo, ECE Dept., Dec. 2006, available at www.cst.uwaterloo.ca.

[4] P. Gupta and P. R. Kumar, "The capacity of wireless networks," IEEE Trans. on Inf. Theory, vol. 46, no. 2, pp. 388 - 404, March 2000.

[5] M. Grossglauser and D. Tse, "Mobility increases the capacity of ad-hoc wireless networks," in Proc. IEEE INFOCOM, April 2001, pp. 1360 - 1369.

[6] S. R. Kulkarni and P. Viswanath, "A deterministic approach to throughput scaling in wireless networks," IEEE Trans. on Information Theory, vol. 50, no. 6, pp. 1041 - 1049, June 2004.

[7] L.-L. Xie and P. R. Kumar, "A network information theory for wireless communication: scaling laws and optimal operation," IEEE Trans. on Information Theory, vol. 50, no. 5, pp. 748 - 767, May 2004.

[8] R. Gowaikar, B. Hochwald, and B. Hassibi, "Communication over a wireless network with random connections," IEEE Trans. on Information Theory, vol. 52, no. 7, pp. 2857-2871, July 2006. 
[9] M. Sharif and B. Hassibi, "A delay analysis for opportunistic transmission in fading broadcast channels," in Proc. IEEE INFOCOM, March 2005.

[10] N. Bansal and Z. Liu, "Capacity, delay and mobility in wireless ad-hoc networks," in Proc. IEEE INFOCOM, April 2003, pp. 1553 - 1563.

[11] S. Toumpis and A. J. Goldsmith, "Large wireless networks under fading, mobility, and delay constraints," in Proc. IEEE INFOCOM, March 2004.

[12] A. El Gamal, J. Mammen, B. Prabhakar, and D. Shah, "Optimal throughput-delay scaling in wireless networks - part I: The fluid model," IEEE Trans. on Information Theory, vol. 52, no. 6, pp. 2568-2592, June 2006.

[13] G. Sharma, R. R. Mazumdar, and N. B. Shroff, "Delay and capacity trade-offs in mobile ad hoc networks: a global perspective," in Proc. IEEE INFOCOM. Barcelona, Spain, April 2006.

[14] M. J. Neely and E. Modiano, "Capacity and delay tradeoffs for ad hoc mobile networks," IEEE Trans. on Information Theory, vol. 51, no. 6, pp. 1917-1937, June 2005.

[15] C. Comaniciu and H. V. Poor, "On the capacity of mobile ad hoc networks with delay constraints," IEEE Trans. on Wireless Commun., vol. 5, no. 8, pp. 2061-2071, August 2006.

[16] M. J. Neely, "Order optimal delay for opportunistic scheduling in multi-user wireless uplinks and downlinks," in Proc. of 44th Allerton Conference on Communication, Control, and Computing, Sept. 2006.

[17] D. E. Knuth, "Big omicron and big omega and big theta," in ACM SIGACT News, April-June 1967, vol. 8 , pp. $18-24$.

[18] P. Viswanath, D. N. C. Tse, and R. Laroia, "Opportunistic beamforming using dumb antennas," IEEE Trans. on Information Theory, vol. 48, no. 6, pp. 1277-1294, June 2002. 